\title{
Labyrinth-based left-handed metamaterials and subwavelength focusing of electromagnetic waves
}

Ekmel Ozbay

Irfan Bulu

Humeyra Caglayan 


\title{
Labyrinth based left-handed metamaterials and sub-wavelength focusing of electromagnetic waves
}

\author{
Ekmel Ozbay $^{a}$, Irfan Bulu ${ }^{a}$, and Humeyra Caglayan ${ }^{a}$ \\ ${ }^{a}$ Department of Physics and Nanotechnology Research Center, Bilkent University, Bilkent, \\ 06800 Ankara, Turkey;
}

\begin{abstract}
We propose and demonstrate a resonant structure that solves two major problems related to the split-ring resonator structure. These major problems may be stated as the bianisotropy and electric coupling to the magnetic resonance. These two problems introduce difficulties in obtaining isotropic left-handed metamaterial mediums. The resonant structure that we propose here solves both of these problems. We further show that in addition to the magnetic resonance, when combined with a suitable wire medium, the structure that we propose exhibits left-handed transmission band. We further demonstrate the sub-wavelength focusing of electromagnetic waves by use of a two dimensional labyrinth based metamaterial. Our experimental results showed that it is possible to focus the source field with half widths as small as $\lambda / 4$ by using the labyrinth based metamaterial.
\end{abstract}

Keywords: Metamaterial, Magnetic Resonance, Split-ring Resonator, Sub-wavelength Focusing, Left-handed medium

\section{INTRODUCTION}

The possibility of the negative refraction of electromagnetic (EM) waves by materials with simultaneous negative permittivity and negative permeability was predicted by Vesalago in $1968 .{ }^{1}$ This proposition was not demonstrated until recently. The missing ingredient was a medium that possess negative permeability. The lack of the negative permeability medium was remedied by Pendry et. al.. Pendry proposed several structures in order to obtain negative permeability. ${ }^{2}$ Among the structures proposed by Pendry et. al., split-ring resonator structure attracted much attention and have been widely used to obtain negative index medium. ${ }^{3-8}$

A single SRR is composed of two concentric rings with slits on each of them. The slits on the rings are situated on the opposite sides of the rings with respect to each other. The planar nature of the SRR structure makes it easy to fabricate and integrate into 2 and 3 dimensional structures. While SRR structure provides negative permeability and can be used to obtain negative refraction, it has several disadvantages. First of all, it has been shown that a medium consisting of a periodic arrangement of SRRs is bianisotropic. ${ }^{9-11}$ The bianisotropy is a result of the non-zero electric dipole moment of the SRR structure due to the asymmetric placement of slits on the rings. Second, it has been shown that the magnetic resonance of the SRR structure can be excited via electric fields. ${ }^{12}$ The excitation of the magnetic resonance of the SRR structure results from the capacitive coupling of the electric field. The capacitive coupling of the electric field creates non-zero current along the rings. These two disadvantages make it difficult to obtain isotropic, homogeneous two or three dimensional negative refraction media by using SRRs for negative permeability.

We propose a new structure to solve the problems outlined above. The proposed structure is more symmetric when compared to the SRR structure. We theoretically and experimentally show that the proposed structure does not exhibit bianisotropy. We show that magnetic resonance of the proposed structure cannot be excited by incident electric fields. In addition, we demonstrate that the periodic arrangement of the proposed resonator and wires exhibit simultaneous negative permittivity and permeability over a frequency range. We further demonstrate the sub-wavelength focusing of electromagnetic waves emitted from a source by use of a two dimensional metamaterial, which was based on the proposed structure.

Further author information: (Send correspondence to E. Ozbay)

E-mail: ozbay@fen.bilkent.edu.tr

Photonic Crystal Materials and Devices IV, edited by Ali Adibi, Shawn-Yu Lin, Axel Scherer,

Proc. of SPIE Vol. 6128, 612813, (2006) · 0277-786X/06/\$15 · doi: 10.1117/12.649548 


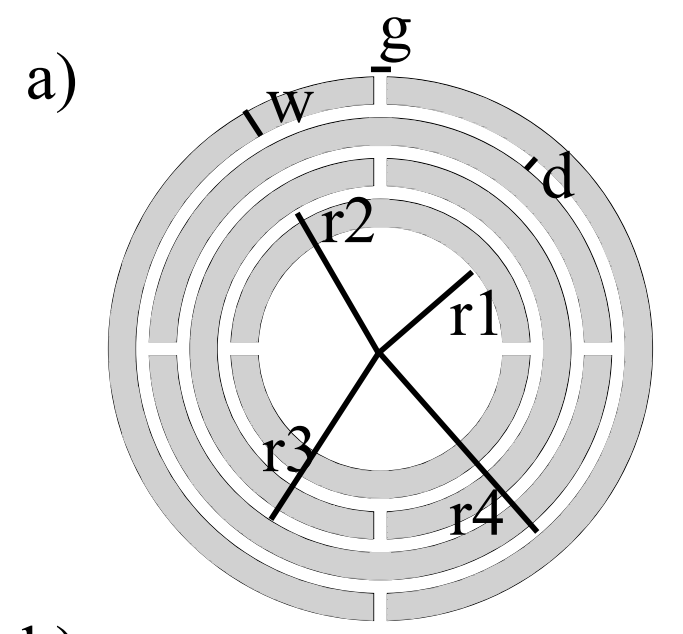

b)

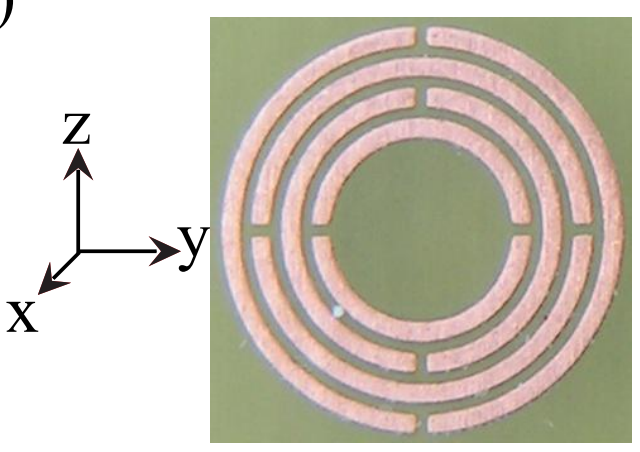

Figure 1. (Color online) a) Schematics of the labyrinth structure. $r 1=1.35 \mathrm{~mm}, r 2=1.8 \mathrm{~mm}, r 3=2.25 \mathrm{~mm}, r 4=2.7$ $\mathrm{mm}, g=0.15 \mathrm{~mm}, w=0.3 \mathrm{~mm}$, and $d=0.15 \mathrm{~mm}$. b) The unit cell of the actual, fabricated structure and the coordinate system that we use throughout the paper.

\section{AN IMPROVED MAGNETIC RESONATOR STRUCTURE FOR NEGATIVE PERMEABILITY: THE LABYRINTH STRUCTURE}

Figure 1(a) shows schematics of the modified SRR structure that we propose. We call the modified SRR as "labyrinth" structure due to its shape. The labyrinth structure consists of four rings instead of two. The two additional rings are used for two main purposes, of which the first is to enhance the strength of the resonance. Second, the two-ring structure has two magnetic resonances that are close to each other. We used the additional two rings in order to separate the two magnetic resonances further away in frequency from each other. The unit cell of the fabricated structure is shown in Fig. 1(b). The structures are fabricated by using standard printed circuit board manufacturing methods. Figure 1(b) also shows the coordinate system that we used throughout the entire paper.

We calculated the induced surface currents, electric field distributions, and transmission properties of incident plane EM waves through the labyrinth structure by using a commercial 3 dimensional full-wave solver. We also measured the transmission properties of the labyrinth structure. The transmission properties of a single labyrinth structure are measured by using a HP 8510C vector network analyzer and two monopole antennas as receiver and transmitter antennas. The measured and calculated transmission spectrum of plane EM waves through a single layer of labyrinth structure is shown in Fig. 2(a). The directions of the electric field, magnetic field, and wave vector of the incident EM waves are shown in Fig. 2(b). First of all the transmission spectrum exhibits a resonance around $6.2 \mathrm{GHz}$ with a transmission of $-25 \mathrm{~dB}$. Second, the closed labyrinth structure does not exhibit this resonance in the transmission spectrum. The calculated induced surface current is shown in Fig. 2(b). The 
a)

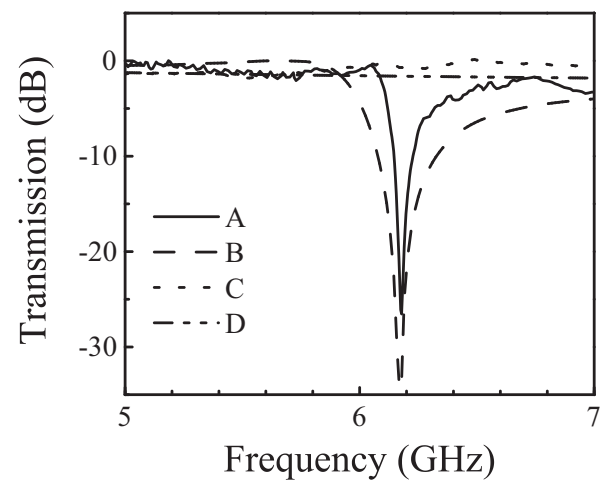

c)

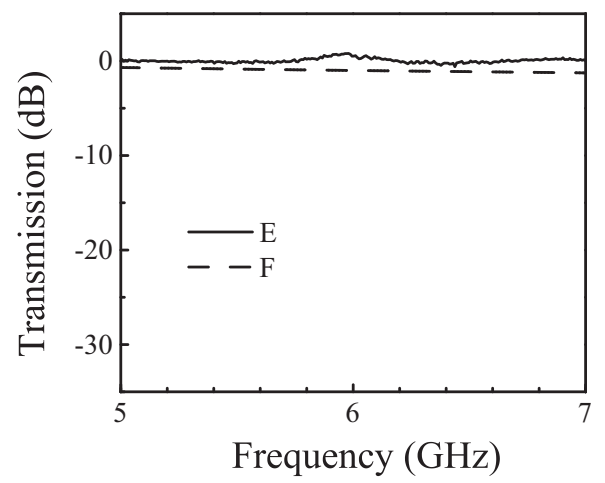

b)

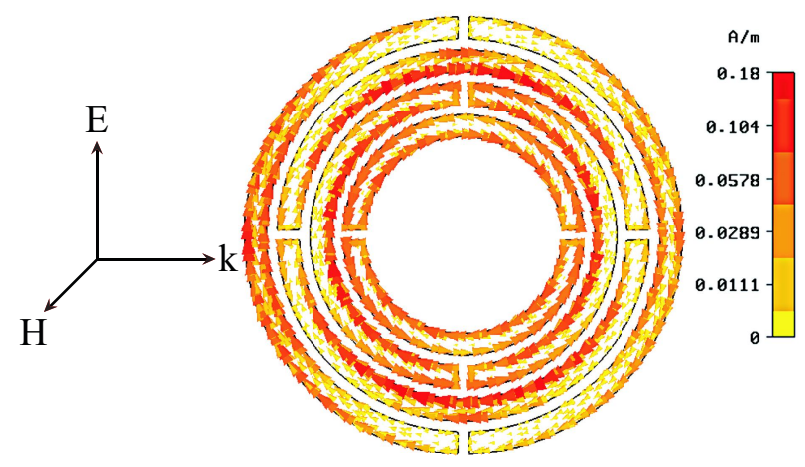

d)

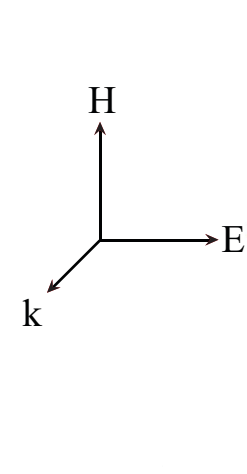

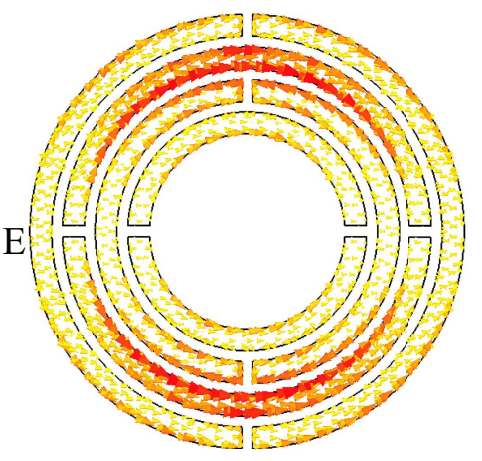

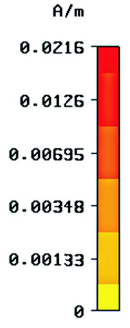

Figure 2. a) Measured transmission through a single labyrinth structure (A), a single closed labyrinth structure (B). Calculated transmission through a single labyrinth structure (C), a single closed labyrinth structure (D). b) Induced surface current density at $6.2 \mathrm{GHz}$. c) Measured (E) and calculated (F) transmission through a single labyrinth structure. d) Induced surface current density at $6.2 \mathrm{GHz}$.

induced surface currents are solenoidal and in phase along each arm. As a result the induced dipole moment has a magnetic character. The comparison of the transmission spectrum of the labyrinth structure with the transmission spectrum of the closed labyrinth supports this conclusion as the closed labyrinth structure does not exhibit any resonance near $6.2 \mathrm{GHz}$. Hence, a single labyrinth structure with the given dimensions exhibits magnetic resonance around $6.2 \mathrm{GHz}$.

The magnetic resonance of the SRR structures can be excited by incident waves whose electric field is perpendicular to the slits and whose wave vector is perpendicular to the plane of SRRs. ${ }^{12}$ Such an incident electric field couples to the magnetic resonance capacitively and induces currents flowing across the rings. The induced currents on both rings are solenoidal, hence they resemble the currents that are induced by incident magnetic fields at the magnetic resonance. The resonance frequency observed due to the capacitive coupling of electric field is quite close to the magnetic resonance. The transmission spectra of the EM waves with magnetic fields perpendicular to the plane of SRRs and with wave vectors in the plane of SRRs are quite close to the transmission spectrum of electromagnetic waves with electric field perpendicular to the slits and with wave vector perpendicular to the plane of SRRs. Hence, the excitation of the magnetic resonance via incident electric fields introduces a problem if one attempts to obtain isotropic and homogeneous negative refraction media by using SRRs and wires.

In order to check whether the magnetic resonance of the labyrinth structure may be excited by incident 
electric fields, we measured and calculated transmission spectrum through a single layer of labyrinth structure when the wave vector is in to the plane of labyrinth structure. The directions of the electric field, magnetic field, and wave vector of the incident EM waves are shown in Fig. 2(d). Note that for the case of incidence configuration shown in Fig. 2(d) the magnetic resonance of the SRR structure can be excited by electric fields. The measurement and calculation results for a single labyrinth structure are shown in Fig. 2(c). The transmission spectrum does not show any resonance around $6.2 \mathrm{GHz}$. In addition, the surface current density that we obtained through our calculations is shown in Fig. 2(d). First of all, the surface current density is reduced by an order of magnitude when compared to Fig. 2(b). Second, the surface current density along each arm of the labyrinth structure is balanced either by an opposite surface current density on the same arm or by an opposite surface current density flowing along the opposite direction on the opposite arm. As a result, the transmission spectrum shown in Fig. 2(c) and the surface current density shown in Fig. 2(d) clearly demonstrate that the magnetic resonance of the labyrinth structure cannot be excited by incident electric fields.

Several researchers have pointed out the bianisotropy issue related to the SRR structure and they have suggested several ways to overcome it. ${ }^{9-11}$ One such way is to place rings with equal radiuses on the opposite sides of the substrate. ${ }^{11}$ In this case the currents flowing across the slits are equally balanced and as a result the bianisotropy is greatly reduced. This approach solves the problem related to the SRR metamaterial medium. But if one desires to combine this modified SRR metamaterial medium with a wire medium in order to obtain negative refraction they face a manufacturing problem. The placement of the wires is not obvious in this case. In addition, this approach cannot solve the problem related to the excitation of the magnetic resonance via incident electric waves.

For the case of incidence depicted in Fig. 2(b), the SRR structure exhibits bianisotropy near the first magnetic resonance i.e., one also observes a non-zero electric field component parallel to the incident magnetic field in the transmission spectrum. In order to demonstrate that the labyrinth structure does not exhibit bianisotropy, we measured the transmission spectrum through a labyrinth metamaterial medium. The transmission measurements were performed with a HP-8510C network analyzer by using horn antennas as the receiver and transmitter. The labyrinth metamaterial is composed of periodic arrangement of labyrinths in a 1 dimensional array of 25 layers along the $\mathrm{x}$ direction, 20 layers along the $\mathrm{z}$ direction, and 5 or 10 layers along the propagation direction (y-axis). The directions are those of Fig. 1(b). The incident electric field is along the $\mathrm{z}$ direction and the wave vector is parallel to the $\mathrm{y}$ direction. We measured both the $\mathrm{x}$ and $\mathrm{z}$ components of the transmitted electric fields. Note that the incident magnetic field is parallel to the $\mathrm{x}$-axis. The transmission measurement results for the $\mathrm{z}$ component of the electric field are shown in Fig. 3(a). The measured transmission data for the medium composed of closed labyrinth structures with the same number of layers is also shown in Fig. 3(a). The transmission spectrum for the labyrinth metamaterial medium exhibits a band gap between $5.9 \mathrm{GHz}$ and $6.6 \mathrm{GHz}$ for the z component of the electric field. The transmission spectrum for the closed labyrinth structure does not exhibit such a band gap. More importantly, we did not detect any appreciable electric field along the $\mathrm{x}$ direction in the transmission spectrum of the labyrinth metamaterial medium (Figure 3(b)). The x component of the electric field is measured by rotating the receiver horn antenna by 90 degrees. We measured the $\mathrm{x}$ component of the electric field with and without the labyrinth metamaterial medium in between the transmitting and receiving horn antennas. The transmitted $x$ component of the electric field is around $-40 \mathrm{~dB}$ within the frequency range of interest in free space. Note that the polarization of the transmitting horn antenna is such that the emitted electric fields are $\mathrm{z}$ polarized. The measured transmission coefficients of the $\mathrm{x}$ component of the electric fields drop below $-40 \mathrm{~dB}$ when the labyrinth structure is inserted between the horn antennas. Hence, these results clearly show that the labyrinth metamaterial medium is not bianisotropic.

\section{LABYTRINTH BASED METAMATERIAL MEDIUM AND FOCUSING OF ELECTROMAGNETIC WAVES}

The composite metamaterial medium (CMM) structure that we used in our study was composed of onedimensional periodic arrangement of labyrinth structures and wire structures. Wires were printed on the back surface of the PCBs and labyrinth structures were fabricated on the front surface of the PCBs. The width of the wire stripes was chosen to be $2.5 \mathrm{~mm}$. This choice was made in order to obtain a plasma frequency at a far enough frequency from the magnetic resonance of the labyrinth structure. The length of the wire stripes was 

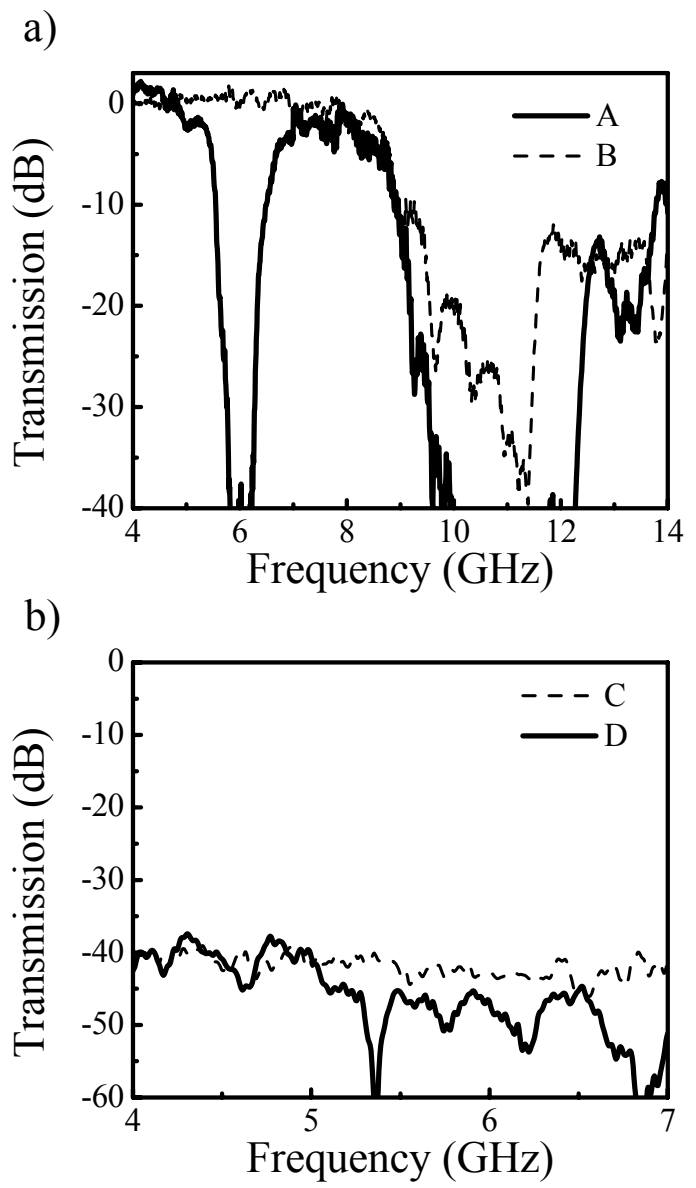

Figure 3. a) Measured transmission spectrum of the z-component of the electric field through (A) the labyrinth metamaterial medium and (B) through the closed labyrinth metamaterial medium. Only the z-component of the incident electric field was nonzero. b) Measured transmission spectrum of the x-component of the electric field through (C) free space and through (D) the labyrinth metamaterial medium. Only the z-component of the incident electric field was nonzero. 

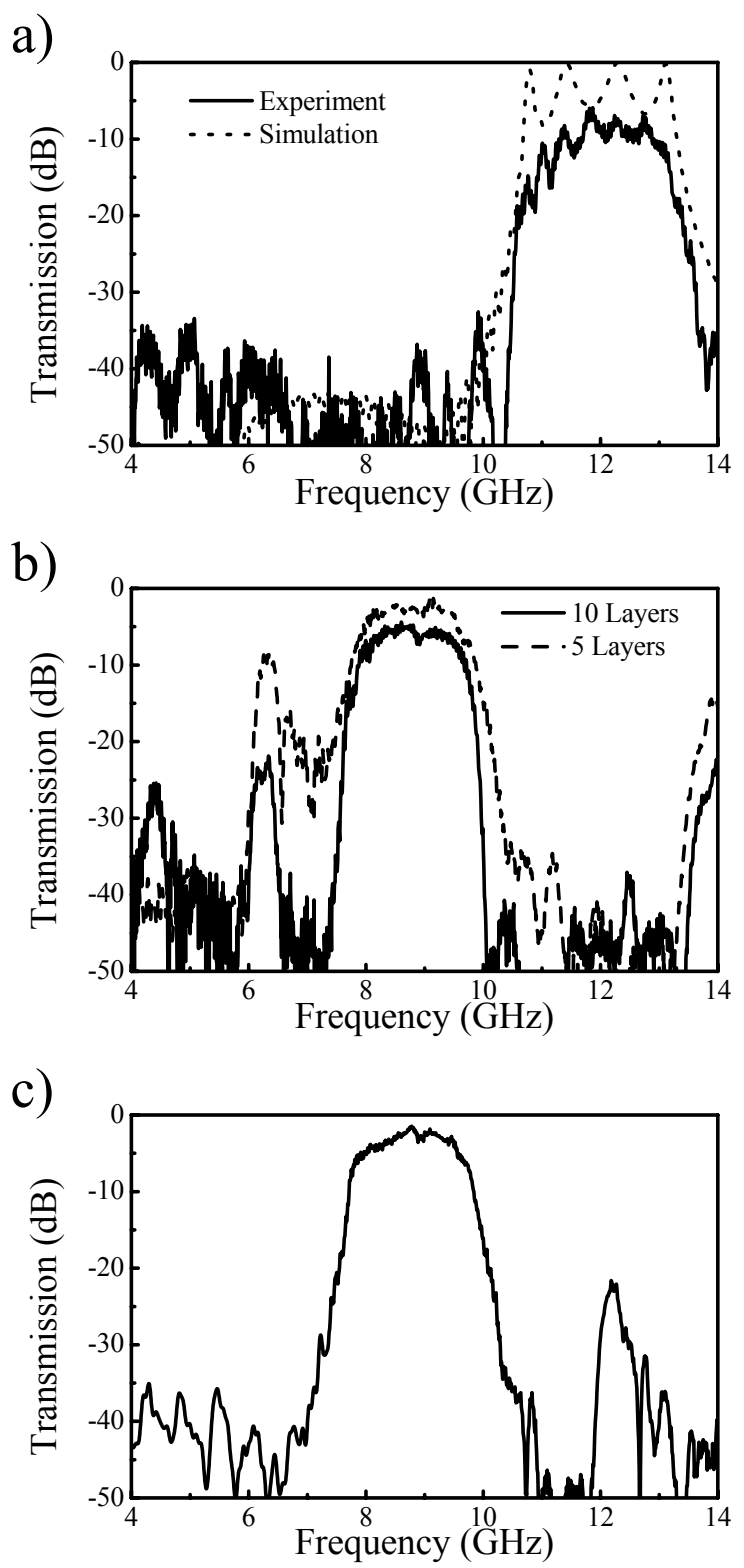

Figure 4. a) Transmission spectrum of electromagnetic waves through the wire medium. b) Measured transmission spectrum of electromagnetic waves through the CMM medium. c) Measured transmission spectrum of electromagnetic wave through the closed CMM medium. 


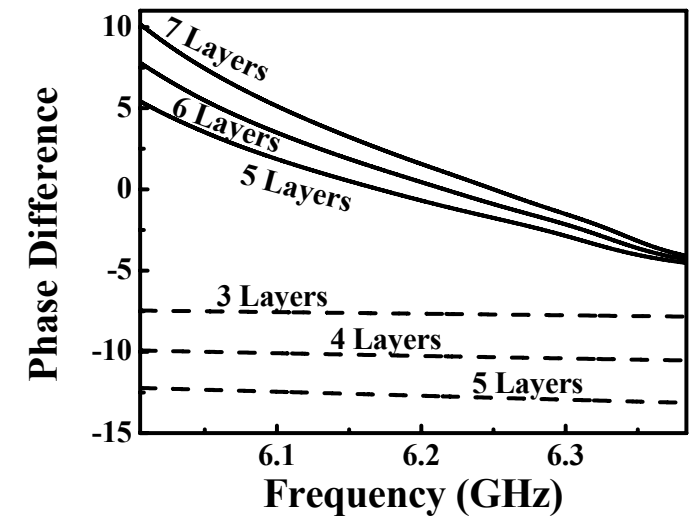

(a)

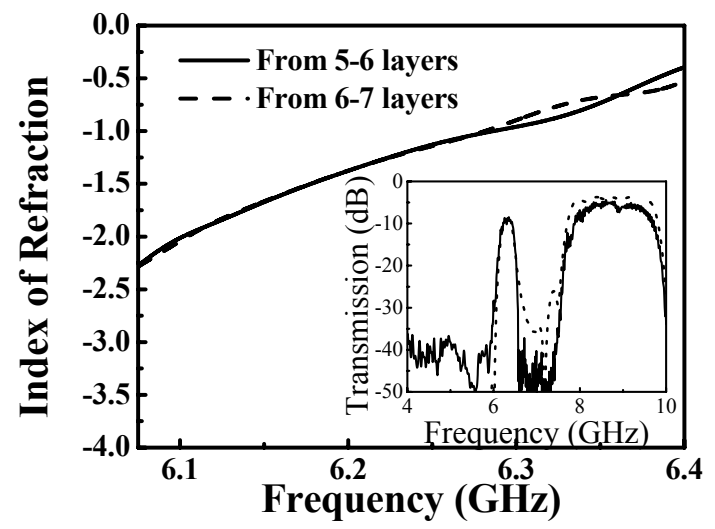

(b)

Figure 5. a) Phase differences between the ends of isotropic FR4 slabs (dashed curves) and the labyrinth based metamaterial (solid curves) b) Calculated indices of refraction for the labyrinth based metamaterial. (inset: measured transmission spectrum through the 5 layer 2D labyrinth based composite metamaterial medium (solid-curve) and the simulated transmission spectrum (dotted-curve).)

$17.6 \mathrm{~cm}$ and the thickness of the stripes was $0.05 \mathrm{~mm}$. The periodic arrangement of wire stripes had a lattice constant of $8.8 \mathrm{~mm}$ along $\mathrm{y}$-axis and $6.5 \mathrm{~mm}$ along $\mathrm{x}$-axis. The propagation direction was along $\mathrm{y}$-axis. There were 10 layers of wire stripes along the y direction and 25 layers along the $\mathrm{x}$ direction. Measured transmission spectrum of the wire medium is shown in Fig. 4(a). The transmission spectrum for the wire medium exhibits a forbidden frequency range of up to $10.45 \mathrm{GHz}$. The plasma edge $(10.45 \mathrm{GHz})$ of the wire medium is $4.2 \mathrm{GHz}$ above the magnetic resonance of the labyrinth structure.

For the CMM structure, wires and labyrinth structures were aligned such that the axis of the wires were parallel to the splits on the labyrinth structure. There were 20 layers of CMM unit cells along z-axis and 25 layers of CMM unit cells along x-axis. The transmission spectrum for 5 and 10 layers of CMM unit cells along the propagation direction is shown in Fig. 4(b). Figure 4 shows that the transmission spectrum of the CMM medium exhibits a transmission band between $5.9 \mathrm{GHz}$ and $6.55 \mathrm{GHz}$. Note that the magnetic resonance of the single labyrinth structure was observed at $6.2 \mathrm{GHz}$. In addition, the labyrinth structure exhibited a band gap between $5.9 \mathrm{GHz}$ and $6.6 \mathrm{GHz}$. Hence, the transmission band of the CMM structure coincides with the band gap of the labyrinth metamaterial medium. We measured the transmission spectrum of the closed CMM medium in order to check whether the transmission band observed between $5.9 \mathrm{GHz}$ and $6.55 \mathrm{GHz}$ is left-handed. ${ }^{13,14}$ The closed CMM medium consists of a periodic arrangement of closed labyrinth structures and wires stripes. The lattice parameters were kept the same as the CMM medium. The transmission spectrum of the closed CMM medium is shown in Fig. 4(c). First of all, the transmission spectrum of the closed CMM medium did not exhibit 


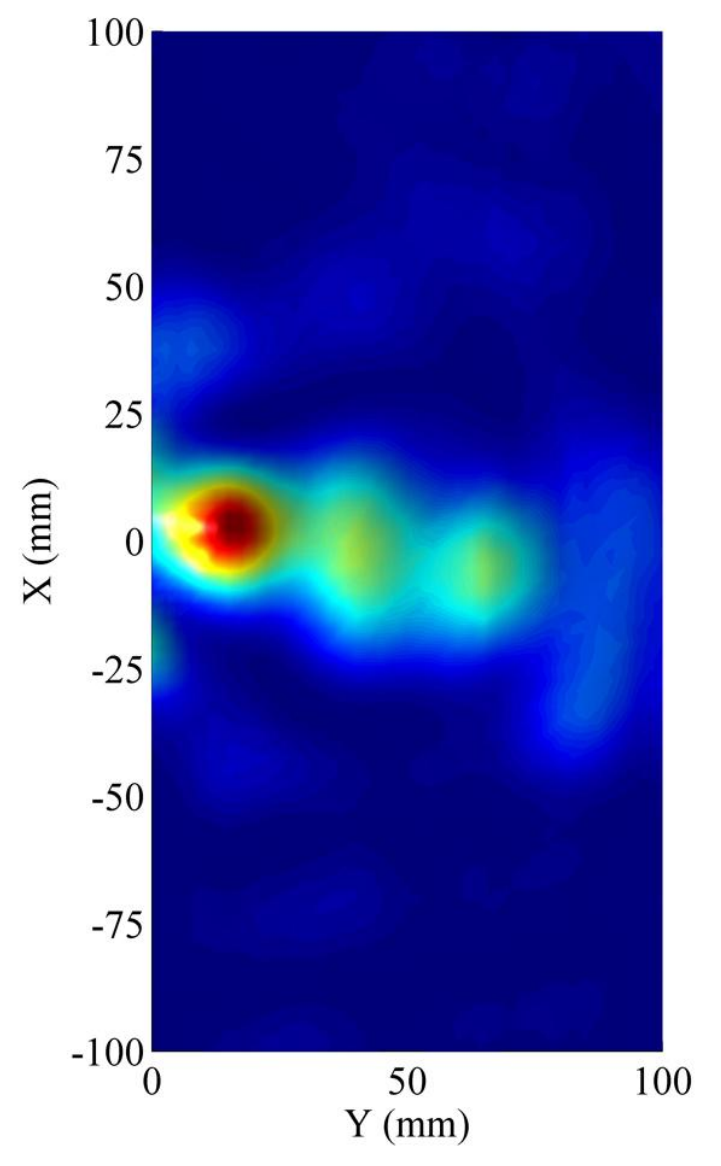

(a)

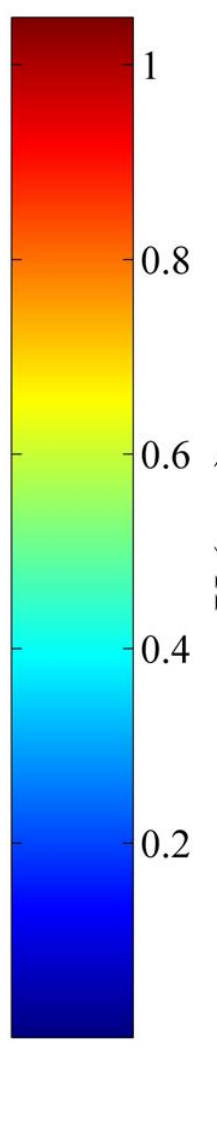

0.2

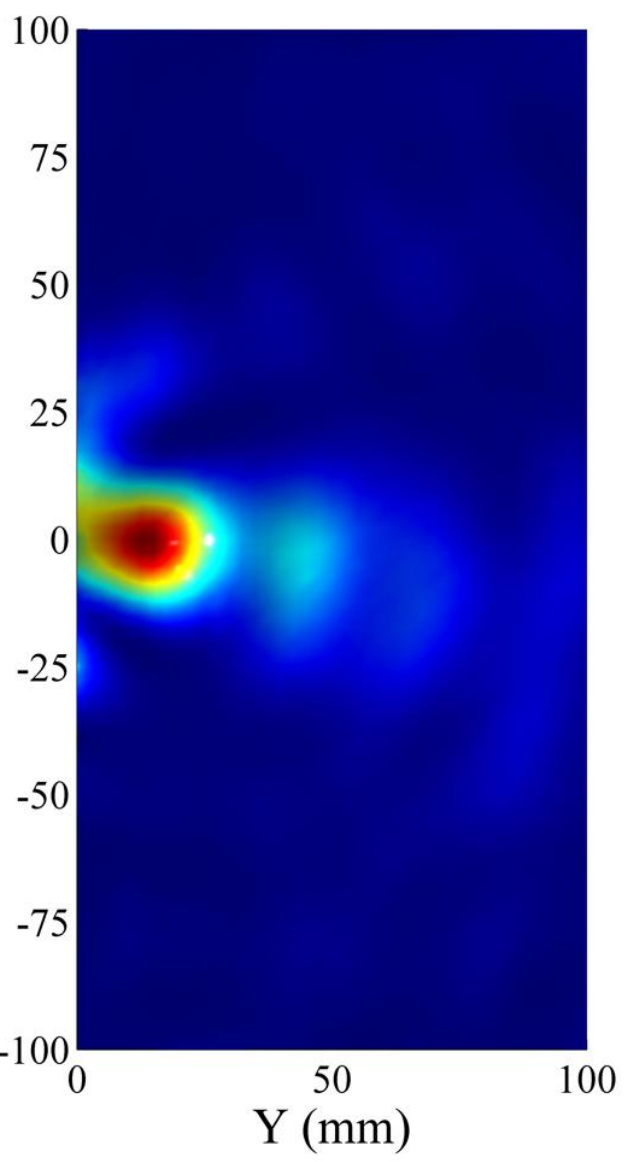

(b)

Figure 6. Measured electric field intensities on the output side of the metamaterial when the source was $2 \mathrm{~cm}$ away (a) and $1 \mathrm{~cm}$ away (b) from the input surface of the metamaterial.

a transmission band between $5.9 \mathrm{GHz}$ and $6.55 \mathrm{GHz}$. These results therefore show that the transmission band of the CMM medium is left-handed. In addition, the transmission spectrum of the closed CMM medium showed that the plasma edge of the wire medium shifts dramatically towards lower frequencies when the wire medium was combined either with a labyrinth medium or closed labyrinth medium. The plasma edge shifted from 10.45 $\mathrm{GHz}$ down to $7.6 \mathrm{GHz}$. Similar results demonstrating the shifting of the plasma edge towards lower frequencies were also reported for metamaterial mediums composed of SRR structures and wire structures. ${ }^{14}$

Several methods, such as retrieval procedures from S-parameters, can be used for the determination of the index of refraction. ${ }^{9}$ Another rather straightforward method makes use of the phase shifts when the size of the structure along the propagation direction is increased. ${ }^{15}$ It was experimentally shown that this method can accurately describe the real part of the index of refraction values for metamaterials even when the transmission was below $-10 \mathrm{~dB} .{ }^{15}$ Consider two pieces of homogeneous material with lengths of $L_{1}$ and $L_{2}$. The phase difference introduced due to the difference in lengths of the pieces can be written as $\Delta \phi=-\mathbf{k}_{0} n\left(L_{2}-L_{1}\right)$, where $\mathbf{k}_{0}$ is the free space wave vector. We used the $-\mathbf{k}_{0}$ convention in this study. In order to theoretically determine the phase shifts when the number of layers along the propagation direction is increased, we performed finiteintegration method simulations by using a commercially available software program. The simulation results for 


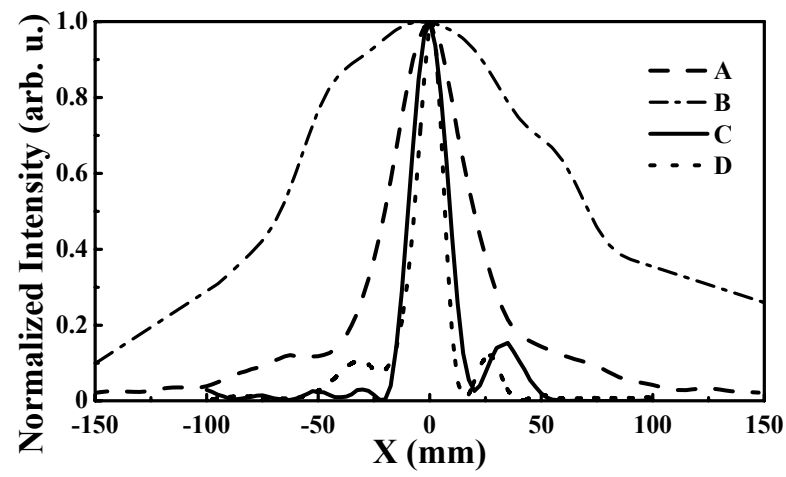

Figure 7. Measured intensity profile of the source monopole antenna along the $\mathrm{x}$ axis in free space when it was placed $2 \mathrm{~cm}$ away from the receiver antenna (A) $8 \mathrm{~cm}$ away from the receiver antenna (B). Measured intensity profile along the $\mathrm{x}$ axis on the output side of the metamaterial when the source was placed $2 \mathrm{~cm}$ away (C) and $1 \mathrm{~cm}$ away (D) away from the input surface of the metamaterial.

the phase differences between the ends of 5 layers, 6 layers, and 7 layers long metamaterials are shown in Fig. 5 (a). For comparison, we plotted the phase differences between the ends of 3 layers, 4 layers, and 5 layers long homogeneous, isotropic FR4 slabs. First of all, note that the phase differences for the FR4 slabs advance in the negative direction when the number of layers is increased, as expected from a medium with positive index of refraction. Whereas, the phase differences between the ends of the metamaterial advance in the positive direction with increasing length along the propagation direction. In addition, the phase differences for different lengths of metamaterials do not change significantly around $6.4 \mathrm{GHz}$. We determined the index of refraction for the metamaterial medium by using the phase shifts shown in Fig. 5 (a). The results between $6.08 \mathrm{GHz}$ and $6.4 \mathrm{GHz}$ are plotted in Fig. 5 (b). The frequency range was chosen in order to have a transmission that was above $-20 \mathrm{~dB}$ for accurate phase determination. The index of refraction is negative within this frequency range. In addition, there is a frequency range around $6.28 \mathrm{GHz}$ at which the index of refraction is close to -1 .

These results show that labyrinth based CMM structure exhibits simultaneous negative permittivity and permeability over a certain frequency range. One fascinating consequence in particular of simultaneous negative permittivity and negative permeability is the possibility of focusing electromagnetic waves beyond the diffraction limit. ${ }^{16}$ Pendry predicted that a slab of $\epsilon=-1, \mu=-1$ may recover evanescent components of the field emitted from a source. In addition, due to negative refraction, such a medium focuses the propagating components of the source field. As a result, it may be possible to focus all of the Fourier components of the field emitted from a source.

In order to study the focusing effect, we placed a source in front of the labyrinth based CMM medium and we checked for the field intensities on the other side of the CMM medium. In our experiments we used monopole antennas as the source and receiver. We placed the source antenna in front of the surface of the labyrinth based metamaterial. The axis of the source and receiver antennas were arranged parallel to the z-axis. The length and diameter of the monopoles were $3 \mathrm{~cm}$ and $1.5 \mathrm{~mm}$, respectively. We measured the electric field intensities over an area of $200 \times 100 \mathrm{~mm}^{2}(4.25 \lambda \times 2.1 \lambda)$ area on the output side of the left-handed metamaterial in steps of 2.5 $\mathrm{mm}(0.052 \lambda)$, where $\lambda$ corresponds to $6.3 \mathrm{GHz}$. The measured electric field intensities when the source antenna was placed $2 \mathrm{~cm}(0.42 \lambda)$ and $1 \mathrm{~cm}(0.21 \lambda)$ away from the input surface of the metamaterial are plotted in Fig. 6 (a) and (b), respectively. The measurement frequency was $6.3 \mathrm{GHz}$. Note that the surface of the metamaterial was parallel to the $\mathrm{x}$ axes. Figures 6 (a) and (b) clearly demonstrate the focusing of the source field on the output side by the left-handed metamaterial when the source was placed either $2 \mathrm{or} 1 \mathrm{~cm}$ away from the input surface. The maximum field intensity along the y axes was observed at $10 \mathrm{~mm}(0.21 \lambda)$ when the source was 2 $\mathrm{cm}(0.42 \lambda)$ away from the input surface, in which it was $17.5 \mathrm{~mm}(0.37 \lambda)$ when the source was $1 \mathrm{~cm}(0.21 \lambda)$ away. The half widths of the intensity profiles along the y axis for both cases were $19 \mathrm{~mm}, \approx \lambda / 2.5$. Moreover, the field intensities decay as $1 / r^{2}$ along the y axes, where $r$ is the distance from the focusing point. There are some extra features appearing on the image. We attribute these extra features to the interference from the waves 
reflected by the laboratory environment. The intensity profiles along the $\mathrm{x}$ axis are shown in Fig. 7. In addition, we plotted the measured intensity profile along the $\mathrm{x}$ axis when the source and receiver antennas were placed $2 \mathrm{~cm}$ apart from each other in free space (dashed-curve in Fig. 7). The measured half width of the intensity profile in free space, without the metamaterial in between, was $40 \mathrm{~mm}, \approx \lambda$. Without the metamaterial, even at such a close distance, the source field could not be resolved with a resolution below the wavelength. On the other hand, the half width of the intensity profile on the output side of the metamaterial along the $\mathrm{x}$ axes was $12 \mathrm{~mm}, \approx \lambda / 4$, when the source was $1 \mathrm{~cm}$ away from the input surface, in which it was $16 \mathrm{~mm}, \approx \lambda / 3$, when the source was $2 \mathrm{~cm}$ away from the input surface. As a result, it was possible to the resolve the source field with a resolution below the wavelength when the metamaterial was inserted in between the source and receiver antennas. The focusing property of the metamaterial was not restricted to $6.3 \mathrm{GHz}$. We observed the focusing effect for a range of frequencies between $6.2 \mathrm{GHz}$ and $6.37 \mathrm{GHz}$, although the focusing was not as sharp as 6.3 $\mathrm{GHz}$. Note that the source and image distances do not add up to the width of the slab (0.92 $\lambda)$. We attribute this to the complex part of the index of refraction.

\section{CONCLUSION}

In summary, we proposed a structure, the labyrinth structure, which exhibits magnetic resonance around 6.2 $\mathrm{GHz}$. The magnetic nature of the resonance was confirmed by comparing the transmission spectrum of the labyrinth structure with that of the closed labyrinth structure. The magnetic nature of the resonance was further confirmed by the strong solenoidal surface currents that flow around the labyrinth structure at the resonance frequency. We experimentally demonstrated that the proposed structure does not show bianisotropy and its magnetic resonance cannot be excited by incident electric fields. As a result, the proposed structure provides important improvements over the common SRR structure. We further showed that the composite metamaterial medium of wires and labyrinth structures has a left-handed transmission band between $5.9 \mathrm{GHz}$ and $6.55 \mathrm{GHz}$.

In addition, we demonstrated that it is in fact possible to focus the electromagnetic waves beyond the limit dictated by diffraction. Our experimental results showed that it was possible to focus the source field with a spot size as small as $\lambda / 4$. We attribute the sub-wavelength focusing to two major reasons. First, the index of refraction is negative. Second, the left-handed metamaterial retains the inhomogeneous plane wave components of the source field. To our knowledge, this is the first experimental demonstration of the sub-wavelength focusing of electromagnetic waves by metamaterials in free space. Previously, sub-wavelength focusing was demonstrated by using a hard-wired L-C circuit network. ${ }^{17}$ We believe that our study may find important applications in areas of imaging. More importantly, our experimental results proved that metamaterials based on the labyrinth structure can focus the electromagnetic waves with half widths smaller than the wavelength.

\section{ACKNOWLEDGMENTS}

This work was supported by the European Union under the projects EU-DALHM, EU NOE-METAMORPHOSE, EU-NOE-PHOREMOST, and TUBITAK-104E090. One of the authors (Ekmel Ozbay) acknowledges partial support from the Turkish Academy of Sciences.

\section{REFERENCES}

1. V. G. Veselago, "The electrodynamics of substances with simultaneously negative values of permittivity and permeability," Sov. Phys. Usp. 10, 509, 1968.

2. J. B. Pendry, A. J. Holden, D. J. Robins, and W. J. Stewart, "Magnetism from conductors and enhanced nonlinear phenomena," IEEE Trans. Microwave Theory Technol. 47, 2075, 1999.

3. P. Markos and C. M. Soukoulis, "Transmission studies of left-handed materials," Phys. Rev. B 65, 033401, 2001.

4. Mehmet Bayindir, K. Aydin, E. Ozbay, P. Marko, and C. M. Soukoulis, "Transmission properties of composite metamaterials in free space," Appl. Phys. Lett. 81, 120, 2002.

5. Philippe Gay-Balmaz and Olivier J. F. Martin, "Electromagnetic resonances in individual and coupled splitring resonators," J. Appl. Phys. 92, 2929, 2002. 
6. D. R. Smith, Willie J. Padilla, D. C. Vier, S. C. Nemat-Nasser, and S. Schultz, "Composite Medium with Simultaneously Negative Permeability and Permittivity," Phys. Rev. Lett. 84, 4184, 2000.

7. Andrew A. Houck, Jeffrey B. Brock, and Isaac L. Chuang, "Experimental Observations of a Left-Handed Material That Obeys Snell's Law," Phys. Rev. Lett. 90, 137401, 2003.

8. Z. G. Dong, S. N. Zhu, H. Liu, J. Zhu, and W. Cao, "Numerical simulations of negative-index refraction in wedge-shaped metamaterials ," Phys. Rev. E 72, 016607, 2005.

9. Xudong Chen, Bae-Ian Wu, Jin Au Kong, and Tomasz M. Grzegorczyk, "Retrieval of the effective constitutive parameters of bianisotropic metamaterials," Phys. Rev. E 71, 046610, 2005.

10. R. Marques, F. Mesa, J. Martel, and F. Medina, "Comparative analysis of edge- and broadside- coupled split ring resonators for metamaterial design - theory and experiments ," IEEE Trans. Antennas Propag. 51, 2572, 2003.

11. R. Marques, F. Medina, and R. Rafii-El-Idrissi, "Role of bianisotropy in negative permeability and lefthanded metamaterials," Phys. Rev. B 65, 144440, 2002.

12. N. Katsarakis, T. Koschny, M. Kafesaki, E. N. Economou, and C. M. Soukoulis, "Electric coupling to the magnetic resonance of split ring resonators," Appl. Phys. Lett. 84, 2943, 2004.

13. T. Koschny, M. Kafesaki, E. N. Economou, and C. M. Soukoulis, "Effective Medium Theory of Left-Handed Materials," Phys. Rev. Lett. 93, 107402, 2004.

14. Koray Aydin, Kaan Guven, Maria Kafesaki, Lei Zhang, Costas M. Soukoulis, and Ekmel Ozbay, "Experimental observation of true left-handed transmission peaks in metamaterials ," Opt. Lett. 29, 2623, 2004.

15. K. Aydin, K. Guven, C. M. Soukoulis, and E. Ozbay Appl. Phys. Lett. 86, 124102, 2005.

16. J.B. Pendry, Phys. Rev. Lett. 85, 3966, 2000.

17. A. Grbic, and G. V. Eleftheriades, Appl. Phys. Lett. 82, 1815, 2003. 